\title{
Peripheral Neuropathy Presenting as Myopathy That was Confused With Inflammatory Myositis
}

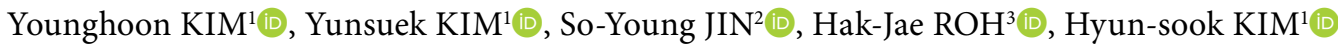 \\ ${ }^{1}$ Department of Internal Medicine, Soonchunhyang University, Seoul, South Korea \\ ${ }^{2}$ Department of Pathology, Soonchunhyang University, Seoul, South Korea \\ ${ }^{3}$ Department of Neurology, Soonchunhyang University, Seoul, South Korea
}

Myopathy accompanying neuropathy is extremely rare. Clinical features alone may be insufficient to distinguish myopathy from neuropathy. ${ }^{1}$ Electrodiagnostic studies such as needle electromyography (EMG), nerve conduction studies (NCS), and muscle biopsies are needed to confirm the diagnosis. ${ }^{2,3}$

A 34-year-old female patient presented with sudden weakness and painful swelling of the left lower extremity. She had also developed multiple bullae that progressed to diffuse swelling (Figure 1a). Laboratory tests resulted in lactate dehydrogenase $881 \mathrm{U} / \mathrm{L}$, aspartate transaminase $264 \mathrm{U} / \mathrm{L}$, alanine transaminase $102 \mathrm{U} / \mathrm{L}$, creatine kinase 23,535 U/L, myoglobin >3,000 (normal 27-75) ng/UL, and aldolase 122 U/L. Antinuclear, anti-Jo antibody, cytomegalovirus, and Epstein-Barr virus were all negative. Magnetic resonance imaging demonstrated general edema and changes in signal intensity around the posterior segment muscles including the semitendinosus and adductor magnus muscles of the left thigh (Figure 2a, b), but it showed no change in the signal intensity of the left calf muscle (Figure 2c). Initially, there were no specific
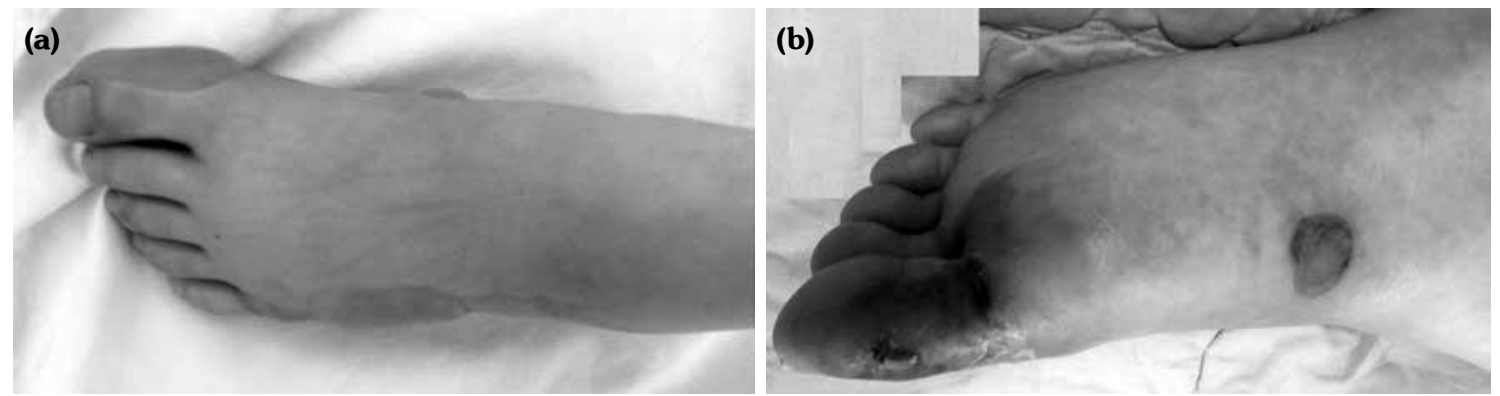

Figure 1. (a) At time of admission, bullae were observed on left foot, with largest measuring $3 \times 4 \mathrm{~cm}$. (b) After one week of glucocorticoid treatment, bullae had revolved almost completely.

Received: December 12, 2017 Accepted: January 13, 2018 Published online: March 23, 2018

Correspondence: Hyun-sook Kim, MD. Department of Internal Medicine, Soonchunhyang University, 04414 Seoul, South Korea. Tel: 821062586498 e-mail: healthyra@schmc.ac.kr 

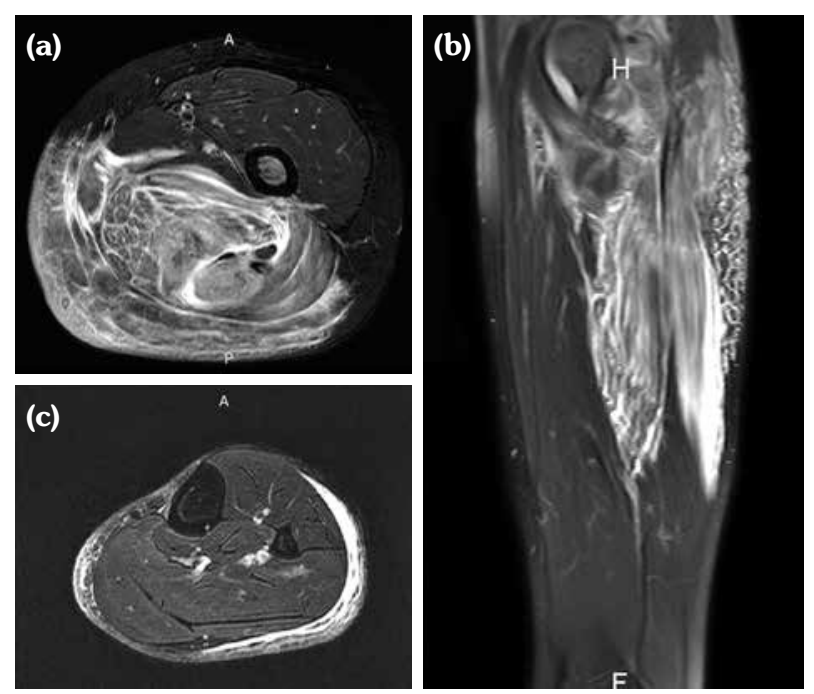

Figure 2. (a, b) $T_{2}$-weighted fat-saturated magnetic resonance imaging revealed diffuse high signal intensity in posterior segmental muscles and subcutaneous fat layer of left thigh. (c) $\mathrm{T}_{2}$-weighted fat-saturated magnetic resonance imaging revealed high signal intensity in subcutaneous fat layer, but not in muscle layer of left calf.

findings of peripheral neuropathy on EMG or NCS. Despite improvement of bullae, edema, and returning to normal range of muscle enzyme after the glucocorticoid pulse therapy (methylprednisolone $250 \mathrm{mg} /$ day for three days) (Figure 1b), the histological findings of the left semitendinosus muscle were nonspecific without interstitial lymphocyte infiltration (Figure 3). Although the patient could extend the left great toe, the left foot plantar flexion and dorsiflexion

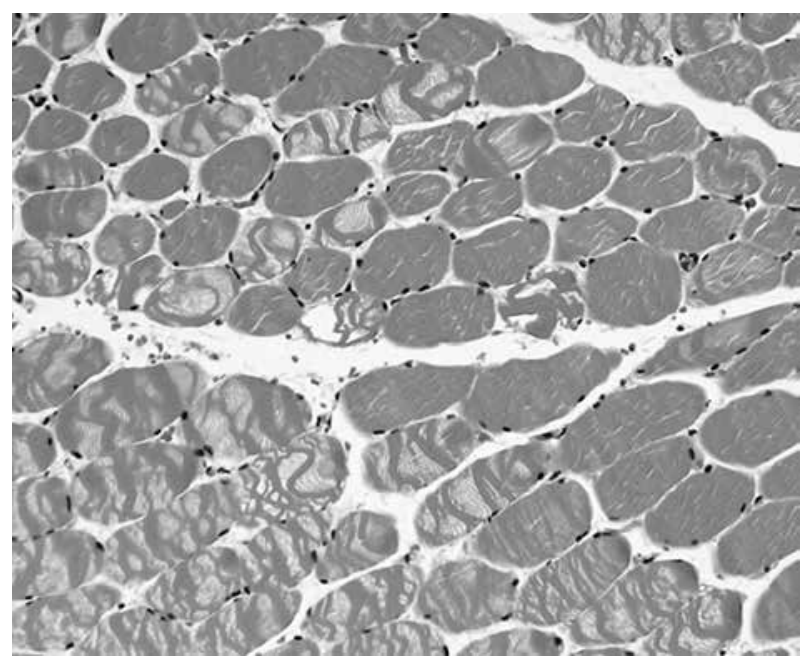

Figure 3. Histologically, left semitendinosus muscle did not change in overall contour or size of muscle fibers, nor was there any central movement of nucleus. Varying degrees of vesicle degeneration and local loss of striation appeared in myocyte cytoplasm. There was no size diversity, mass atrophy, or fat infiltration, and no interstitial lymphocyte infiltration $(\mathrm{H}-\mathrm{E} \times 200)$.

Table 1. There were no signs suggestive of myopathy or neuropathy on needle electromyography at the time of admission. However, three weeks after admission, the needle electromyography findings revealed decreased motor unit activation in the muscles innervated by the peroneal and tibial nerves (the external hallucis longus, tibialis anterior, tibialis posterior, and peroneus longus muscles). After treatment, axonal degeneration and regeneration were observed and the motor unit activation was improved in the same muscles.

\begin{tabular}{|c|c|c|c|c|c|c|c|c|c|}
\hline \multirow[t]{2}{*}{ Muscle (left) } & \multicolumn{3}{|c|}{3 weeks after admission } & \multicolumn{3}{|c|}{9 weeks after admission } & \multicolumn{3}{|c|}{7 months after admission } \\
\hline & ASA & MUAP & IP & ASA & MUAP & $\overline{\text { IP }}$ & ASA & MUAP & IP \\
\hline External hallucis longus & - & - & Z & + & PPP & S-P & - & Normal & P-C \\
\hline Tibialis anterior & - & - & $\mathrm{Z}$ & + & PPP & $\mathrm{P}$ & + & PPP & P-C \\
\hline Tibialis posterior & - & - & Z & + & Normal & $\mathrm{P}-\mathrm{C}$ & - & Normal & $\mathrm{P}$ \\
\hline Peroneus longus & - & - & Z & + & PPP & $\mathrm{P}$ & - & PPP & $\mathrm{P}-\mathrm{C}$ \\
\hline Gastrocnemius (medial) & - & PPP & S-P & + & PPP & $\mathrm{P}$ & - & Normal & $\mathrm{C}$ \\
\hline Biceps femoris short & - & PPP & $\mathrm{C}$ & ++ & PPP & $\mathrm{C}$ & - & Normal & $\mathrm{C}$ \\
\hline Semitendinosus & - & Normal & $\mathrm{C}$ & - & Normal & $\mathrm{C}$ & - & Normal & $\mathrm{C}$ \\
\hline Semimembranosus & - & PPP & $\mathrm{C}$ & - & Normal & $\mathrm{C}$ & - & Normal & $\mathrm{C}$ \\
\hline Biceps femoris long & - & Normal & $\mathrm{C}$ & ++ & Normal & $\mathrm{C}$ & - & Normal & $\mathrm{C}$ \\
\hline
\end{tabular}


Table 2. Nerve conduction study findings revealed decreased amplitude of the left peroneal and tibial nerves, compared with the right side. These findings suggested axonal loss of the left peroneal and tibial nerves. After treatment, the amplitudes of the left peroneal and tibial nerve were increased and the degree of axonal loss was improved

\begin{tabular}{lccc}
\hline & 3 weeks after admission & 9 weeks after admission & 7 months after admission \\
\hline Left deep peroneal nerve-EDB & No response & No response & $0.5 \mathrm{mV}$ \\
Left deep peroneal nerve-TA & $3.6 \mathrm{mV}$ & $4.3 \mathrm{mV}$ & $5.5 \mathrm{mV}$ \\
Left tibial nerve-AH & $(51 \%$ of right side $)$ & $2.1 \mathrm{mV}$ & $7.3 \mathrm{mV}$ \\
& $(23 \%$ of right side) & $2.7 \mathrm{mV}$ \\
\hline EDB: Extensor digitorum brevis; TA: Tibialis anterior; AH: Abductor hallucis. & & \\
\hline
\end{tabular}

Myopathy and neuropathy are generally considered to be different, although concomitant myopathy and neuropathy are rarely reported, called neuromyositis. ${ }^{4}$ EMG and NCS are useful tools for identifying neuromyositis. ${ }^{5}$ Muscle and nerve biopsies may also be helpful for diagnosing neuromyositis, but further studies are needed to establish the typical histologic findings. Myopathy is rarely accompanied by bullae. If the skin cells are damaged by various causes and their structural function is affected, the cells separate from each other and fluids build up between the separated cells, causing bullae. ${ }^{6,7}$ In our case, a similar mechanism was suggested that the rapid progression of myopathy accompanied by neuropathy caused cutaneous edema and dermoepidermal disruption, resulting in bullae.

Although EMG and NCS are useful tests for distinguishing myopathy and neuropathy, the interpretation of the results may vary depending on the time of the tests. Typically, NCS and EMG reveal pathological findings beginning a few days after the onset of symptoms, but they may appear normal for up to three weeks. Therefore, if unexplained abnormal sensation and disability occur, it is appropriate to repeat EMG and NCS.

\section{Declaration of conflicting interests}

The authors declared no conflicts of interest with respect to the authorship and/or publication of this article.

\section{Funding}

This article was supported by the Soonchunhyang University fund.

\section{REFERENCES}

1. Watson JC, Dyck PJ. Peripheral Neuropathy: A Practical Approach to Diagnosis and Symptom Management. Mayo Clin Proc 2015;90:940-51.

2. Suresh E, Wimalaratna S. Proximal myopathy: diagnostic approach and initial management. Postgrad Med J 2013;89:470-7.

3. Ghaoui R, Clarke N, Hollingworth P, Needham M. Muscle disorders: the latest investigations. Intern Med J 2013;43:970-8.

4. Nguyen TP, Bangert C, Biliciler S, Athar P, Sheikh K. Dermatomyositis-associated sensory neuropathy: a unifying pathogenic hypothesis. J Clin Neuromuscul Dis 2014;16:7-11.

5. Milanov I, Ishpekova B. Differential diagnosis of chronic idiopathic polymyositis and neuromyositis. Electromyogr Clin Neurophysiol 1998;38:183-7.

6. Kakurai $M$, Umemoto $N$, Yokokura $H$, Fujiwara T, Yoneda K, Demitsu T. Unusual clinical features of coma blister mimicking contact dermatitis in rhabdomyolysis: report of a case. J Eur Acad Dermatol Venereol 2006;20:761-3.

7. Kashiwagi M, Ishigami A, Hara $\mathrm{K}$, Matsusue $\mathrm{A}$, Waters B, Takayama M, et al. Immunohistochemical investigation of the coma blister and its pathogenesis. J Med Invest 2013;60:256-61. 